\title{
New insights in amelioration of rhabdomyolysis- induced acute kidney injury by triple effect of Diallyl trisulphide and Sildenafil on apoptosis, NF-KB and NRF-2/HO-1 signaling crosstalk
}

Rania Khalil (D rania742002@yahoo.com )

Delta University for Science and Technology

Ghada S. El-Tanbouly

Delta University for Science and Technology

\section{Research Article}

Keywords: Rhabdomyolysis, Diallyl trisulphide, NRF-2, HO-1, NF-KB, BAX/BCL-2

Posted Date: March 7th, 2022

DOI: https://doi.org/10.21203/rs.3.rs-1251671/v1

License: (c) (1) This work is licensed under a Creative Commons Attribution 4.0 International License.

Read Full License 


\section{Abstract}

Acute kidney injury is a crucial complication of rhabdomyolysis. Glycerol (Gly) is used for induction of rhabdomyolysis in rats. Diallyl trisulphide (DATS) is a natural product from garlic with reported antiinflammatory/antioxidant activities. Sildenafil (Sild) is a phosphodiesterase-5 inhibitor, selected in this study for its experimental protective effects in muscle and kidney injuries. Herein, protective effects of DATS and Sild were investigated in rhabdomyolysis-induced acute kidney injury (RIAKI) model. Rats were separated into five groups: Control (saline, i.p.), Gly (10 ml/kg, i.m.), Gly+Sild (5mg/kg, oral), Gly+DATS (10 and $20 \mathrm{mg} / \mathrm{kg}$, i.p.), and tested $24 \mathrm{hrs}$ after injury. Renal and muscle function tests: Serum BUN, creatinine, total $\mathrm{CK}, \mathrm{CK}-\mathrm{MB}$, in addition to urine creatinine, total protein and albumin were measured. Renal oxidant/antioxidant status and histopathological changes, were also measured. Meanwhile, renal NRF-2, HO-1, IL-1 $\beta$ and IL-6, were assessed by ELISA. Furthermore, inflammation (TNF-a, NF-kBp65, COX2) and apoptosis (BAX/BCL-2) were examined by immunohistochemistry. The study showed that DATS, in a dose-dependent manner improved RIAKI, through reduction of mortality, renal damage, muscle injury, oxidative stress inflammation and apoptosis, in addition to improvement of kidney function and antioxidant defense. DATS prevented nuclear translocation of NF-KBp65 and BAX. Otherwise, DATS elevated nuclear translocation of BCL-2 and expression of NRF-2/HO-1, thus modulating the crosstalk between these pathways. In the meantime, sildenafil, to a reduced extent, monitored the same pathways and proved its muscle/reno-protective actions. Therefore, DATS can be a natural candidate for treatment of acute muscle and kidney injuries, via its verified antioxidant, anti-inflammatory and antiapoptotic potentials.

\section{Introduction}

Acute kidney injury (AKI) is linked to elevated hazard of chronic kidney diseases (CKD). Rhabdomyolysis, one of the main origins of acquired AKI, is the result of diverse circumstances, as severe trauma, crush injuries, and some medications (Goldberg and Dennen 2008, Shi, Xu et al. 2017). Rhabdomyolysis is characterized by skeletal muscle injury, which causes release of cell components into systemic circulation. The released cellular contents resulted in oxidative stress, renal vasoconstriction, tubular obstruction, inflammation and apoptosis which eventually caused nephrotoxicity (Panizo, Rubio-Navarro et al. 2015). Preceding studies confirmed that oxidative stress was originally implicated in the pathogenesis of AKI and contributed in its development (Sureshbabu, Ryter et al. 2015).

In addition, numerous signaling pathways involved in inflammation and cell death responses are triggered. Stimulation of nuclear factor kappa B (NF-kB) signaling, was found to be crucial for the progression of rhabdomyolysis-induced AKI (Huang, Zhou et al. 2017). In addition, many cytokines/chemokines, including interleukin-1 (IL-1), interleukin-6 (IL-6) and tumor necrosis factor-a (TNFa), are elevated in the injured kidney after glycerol (Gly) injection (Singh, Junemann et al. 2012, Geng, Wang et al. 2015). 
On the other hand, nuclear factor erythroid 2-related factor 2 (NRF-2) is reported to have an impact on controlling antioxidant mechanisms, and NF-KB inflammatory pathway, directly, and indirectly through activation of heme oxygenase-1 (HO-1) (Swamy, Rajasekaran et al. 2016). The interplay between NRF-2 and NF-KB has been advocated, in which the absence of NRF-2 with elevated oxidative stress is the clue to the NF-KB-mediated cytokine expression (Saber, Khalil et al. 2019). A pivotal linkage has been recognized between NRF-2 and NF-KB signaling paths and rhabdomyolysis-induced AKI (RIAKI).

The modulation of signals of apoptosis may be a critical tool in the pathogenesis of kidney injury. BCL-2 (B-cell lymphoma-2) family of antiapoptotic and proapoptotic proteins are essential regulators of apoptosis in injury (Qiu, Sinniah et al. 2004, Caro-Gómez, Rosas-Trigueros et al. 2019). In renal cells, injury activates the proapoptotic protein; BAX and reduces the antiapoptotic protein; BCL-2, thus remodelling the BAX/BCL-2 ratio in a remarkable proapoptotic direction. Interestingly, both BAX and BCL2 gene expressions are markedly elevated in regenerating proximal tubule cells (Havasi and Borkan 2011). Thereby, interaction between anti- and pro-apoptotic members of the BCL-2 family is assumed to be vital for repair in damaged proximal tubule cells following rhabdomyolysis. Depending on the specific role of NF-KB in apoptosis, it may regulate the expression of either pro-apoptotic or antiapoptotic members of the Bcl-2 protein family (Shou, Li et al. 2002).

Diallyl trisulphide (DATS) is a natural product extracted from garlic and has anti-inflammatory and antioxidant activities. Moreover, DATS has been described as an active component liable for the antiapoptotic influence of garlic oil (Ma, Huang et al. 2014). Most recently, polysulphide components of garlic has been described to protect against glycerol-induced nephrotoxicity in rats, primarily due to its antioxidant and anti-inflammatory effects (Shang, Cao et al. 2019, Sharma, Kaur et al. 2021). Few citations available in literature suggest an effect of DATS on NF-KB and NRF-2 (Miltonprabu, Sumedha et al. 2017, Liang, Li et al. 2019). Additionally, the relevance of this consequence has never been addressed in models of rhabdomyolysis-induced AKI. Therefore, this study was designed to investigate the role of DATS versus glycerol-induced rhabdomyolysis-induced AKI in rats. Moreover, the potential DATSmediated reno-protection through its effect on BCL-2 family is originally explored in this study.

In this context, Sildenafil (Sild), a distinguished phosphodiesterase-5 (PDE-5) inhibitor, is commonly used for treating erectile dysfunctions and pulmonary hypertension. It also prompted protective properties throughout muscle injury on numerous organs (Savvanis, Nastos et al. 2014, Mohey, Singh et al. 2016, Liang, Li et al. 2019), supporting its likely usefulness as a drug for treatment of muscle injury and can be used in comparison with others in reduced muscle oxidative stress and/or inflammation. In addition, its effect on some experimentally-induced kidney injury models, favored the selection of Sild to be used in this study (Dias, Rodrigues et al. 2014, Khames, Khalaf et al. 2017, Behiry, Rabie et al. 2018)

Hence, this study pursued to examine the possible protective effect of DATS on rhabdomyolysis-induced AKI model in rats, in addition to the prospective modulatory effect of DATS on apoptosis (BAX/BCL-2), NF-KB and NRF-2/HO-1 crosstalk. This may deliver new insights on the effect of DATS as a natural 
product in treatment of muscle and/or acute kidney injuries and compare its actions with the documented muscle protective effect of sildenafil.

\section{Materials And Methods \\ 2.1. Drugs and chemicals}

Diallyl trisulfide (DATS) was purchased from (Abcam, MA, USA, catalog \# ab141926). Glycerol (Gly) was purchased from (EL-Goumhouria Co. for trading medicines, Egypt). These drugs were dissolved in $0.9 \%$ saline.

Sildenafil (Sild) was purchased from (Pfizer, Egypt), and dissolved in distilled water. Other chemicals were of the finest analytical grade.

\subsection{Animals}

Adult male SD rats weighting $250 \pm 20 \mathrm{~g}$ were maintained in a room at standard conditions and were provided food and water ad libitum. Rats were allowed 7 days to adapt to the laboratory environment before the experiments. The study and the animal care were approved by the Institutional Animal Care and Use Committee (IACUC) at Faculty of Pharmacy, Delta University for Science and Technology, Gamasa, Egypt, with approval number (FPDU 150321/5).

\subsection{Rhabdomyolysis-induced AKI rat model and experimental design.}

Rats were assigned randomly into 5 groups $(n=9)$ : Control, received $0.9 \%$ saline i.p.; diseased group Glycerol (Gly), injected once with $50 \%$ glycerol $(10 \mathrm{ml} / \mathrm{kg}$, im). For each rat, the dose was equally divided into the two hind limbs muscles; Sild + Gly, received sildenafil ( $5 \mathrm{mg} / \mathrm{kg}$, orally), one hour prior glycerol injection; DATS $10+$ Gly, received DATS (10 mg/kg, i.p.), one hour prior glycerol injection. DATS $20+$ Gly, received DATS $(20 \mathrm{mg} / \mathrm{kg}$, i.p.), one hour prior glycerol injection. All drug doses are selected according to previous reports (Gurbuz, Kol et al. 2015, Zhang, Zhang et al. 2016). All rats were deprived from water with free access to food for $24 \mathrm{hrs}$ and kept in metabolic cages for collection of urine. Twenty-four hours after injection of glycerol, all the rats were sacrificed under anesthesia. Blood samples were collected from the eye of rats in all experimental groups, then allowed to clot and centrifuged at 3,000 rpm, for 10 min to obtain serum. The serum was separated and divided into aliquots.

The kidneys were rapidly separated, washed and weighed to determine the renal somatic index (RSI). RSI was calculated by dividing kidney weight ( $\mathrm{g}$ ) by final body weight $(\mathrm{g})$ then multiplied by 100 . The left kidney was homogenized in phosphate buffer solution (PBS). Kidney homogenates were centrifuged at $4000 \times \mathrm{g}$ at $4^{\circ} \mathrm{C}$ for $15 \mathrm{~min}$. The supernatant was kept in $-80^{\circ} \mathrm{C}$ for further analysis. The right kidney was fixed in $10 \%$ buffered formalin for histopathological and immunohistochemical analyses.

\subsection{Kidney function tests and rhabdomyolysis markers}


Serum and urinary levels of creatinine (Cr), in addition to urinary levels of total protein and albumin were determined by commercially available colorimetric assay kits from (Diamond, SPINREACT Co., Human, Egypt, respectively).

Also, serum level of blood urea nitrogen (BUN), and the rhabdomyolysis markers: total creatine kinase (CK) and creatine kinase-MB (CK-MB), were investigated by commercially available colorimetric assay kits from (Biomed diagnostic, Egypt). All procedures were executed according to the manufacturer's directions.

\subsection{Estimation of oxidant/antioxidant status}

Colorimetric assay kits were used to measure renal levels of malondialdehyde (MDA), nitric oxide (NO), total antioxidant capacity (TAC), superoxide dismutase (SOD), and reduced glutathione (GSH), according to the manufacturer's instructions. The kits were purchased from (Biodiagnostic company, Egypt, catalog number: MD2529, NO2533, TA2513, SD 25 21, and GR 2511 respectively).

\subsection{Evaluation of the antioxidant-related markers (NRF-2, HO-1) by ELISA assay}

Levels of the antioxidant-related markers: nuclear erythroid related factor 2 (NRF-2) and heme oxygenase1(HO-1) were evaluated in kidney homogenate by sandwich enzyme-linked immunosorbent assay (sandwich ELISA), using commercial kits obtained from (Bioassay Technology Laboratory, Shanghai Crystal Day Biotech Co., Ltd., Shanghai, China), according to manufacturer's instructions.

Brie $\llbracket$, a specific biotinylated antibody reagent was added to the plates, which were filled with the homogenized tissue and incubated at $37^{\circ} \mathrm{C}$ in $\mathrm{CO}_{2}$ for $2 \mathrm{hrs}$. After washing with phosphate-buffered saline (PBS), streptavidin horseradish peroxidase (HRP) solution was added and the plates were incubated for $30 \mathrm{~min}$ at room temperature. Then, absorbance was measured at $450 \mathrm{~nm}$ utlizing a microplate reader.

\subsection{Evaluation of the inflammatory mediators (IL-1 $\beta$, IL-6) by ELISA assay}

The concentration of the inflammatory cytokines (IL-1 $\beta$ and IL-6) was detected in kidney homogenate, by ELISA assay, using commercial kits obtained from (Bioassay Technology Laboratory, Shanghai Crystal Day Biotech Co., Ltd., Shanghai, China), according to manufacturer's instructions, as mentioned before.

\subsection{Immunohistochemical examination}

Antibodies were used to evaluate renal expression of tumor necrosis factor-alpha (TNF- a) (ABclonal Technology, MA, USA, catalog number: A0277) and nuclear factor kappa beta p65 (NF-kB p65) (Bioss antibodies, MA, USA, catalog number: bs-20159R). In addition, antibodies were used to evaluate renal expressions of COX-2 (ABclonal Technology, MA, USA, catalog number: A1253), Bax (Servicebio technology Co., China, catalog number: GB11007-1) and Bcl-2 (Genemed Biotechnologies, Inc., CA, USA, 
catalog number: 61005), using Avidin-Biotin Complex (ABC), immunohistochemical staining protocol, following the manufacturer's directions.

Sections $(4 \mu \mathrm{m})$ of renal tissues were incubated for $20 \mathrm{~min}$ in $0.3 \% \mathrm{H} 2 \mathrm{O} 2$ to block the endogenous peroxidase activity. The section slides were incubated in a $100^{\circ} \mathrm{C}$ water bath for $10 \mathrm{~min}$ in $0.01 \mathrm{M}$ PBS buffer solution, then blocked with $2 \%$ BSA for $30 \mathrm{~min}$. Then, the sections were incubated with the anti-rat antibodies overnight at $4^{\circ} \mathrm{C}$. The slides were washed and then incubated with HRP secondary antibody at $37^{\circ} \mathrm{C}$ for $1 \mathrm{~h}$. The sections were then treated with $D A B$ working solution for 4 min and finally counterstained with Mayer's hematoxylin. Tissue sections were observed, photographed with a microscope (400x magnification) and were semi-quantified. The labeling index of TNF-a, NF-kB p65, COX$2, B A X$ and $B C L 2$, was estimated as score of the ratio of positive cells per total 1000 counted cells in about 5 to 8 high power fields.

\subsection{Histopathological examination of renal tissues}

The right kidneys were separated and fixed with $10 \%$ formaldehyde solution for $48 \mathrm{~h}$. The tissues were embedded in paraffin wax and sliced $(5 \mu \mathrm{m})$. The sections were stained with hematoxylin-eosin (H\&E) and were examined blindly by the pathologist under a microscope (400×magnification). Renal injury was scored semi-quantitatively by a previous reported scoring system (Zhao, Huang et al. 2016) for tubular necrosis, tubular dilation, cast formation, congestion and damaged glomeruli. These criteria were assessed on a scale from 0 to 4 , which ranged from absent (0), mild (1), moderate (2), severe (3), to very severe (4).The sum of scores of histopathological lesions were calculated.

Five random fields for each kidney slide were assessed, and the mean score was expressed as the renal injury score, whereas the values were semi-quantified and expressed as mean \pm SEM.

\subsection{Statistical analysis}

Results are presented as the mean \pm standard error of the mean (S.E.M). Statistical analysis was carried out by GraphPad Prism 5 (GraphPad Software, San Diego, CA, USA). One-way analysis of variance followed by Tukey Kramer's post hoc test was used to compare the measures between the experimental groups. Kruskal-Wallis test followed by Dunn's multiple comparison post-hoc test was used for analysis of mortality, histopathological and histochemical scores of rats. Significance level was set at $p<0.05$.

\section{Results}

\subsection{Effect on mortality and renal somatic index (RSI) in rhabdomyolysis-induced AKI}

Gly treated group showed a significant increase to $44.44 \%$ in mortality, compared to control group. This elevation was decreased markedly to $11.11 \%$ in Sild treated group and no mortality was observed in all DATS treated rats. Otherwise, renal somatic index is elevated in Gly group, compared to control rats, while treatments with sildenafil or DATS maintained normal RSI of rats (Table 1) 


\subsection{Effect on kidney function markers}

As shown in Fig. 1, Glycerol induced a significant increase in renal function tests $(p<0.05)$. Meanwhile, renal function tests were significantly reduced in rats treated with sildenafil, and DATS, compared to Gly group. It was notable that rats treated with DATS $20 \mathrm{mg} / \mathrm{kg}$ exhibited the most promising near normal recovery in most renal functions.

\subsection{Effect on rhabdomyolysis markers}

As shown in Fig. 2, Glycerol produced a significant increase in rhabdomyolysis markers $(\mathrm{p}<0.05)$. At a lower dose, DATS $(10 \mathrm{mg} / \mathrm{kg})$, couldn't reverse the elevated serum levels of total CK and CK-MB, compared to Gly group. Meanwhile, the muscle protective drug, sildenafil, in addition to, the higher dose of DATS $(20 \mathrm{mg} / \mathrm{kg})$, were able to alleviate rhabdomyolysis in rats compared to Gly group.

\subsection{Effect on oxidant/antioxidant status}

As shown in Fig. 3, Glycerol induced a significant increase in lipid peroxidation and nitrosative stress, indicated by the elevated renal MDA content and renal NO, respectively, compared to control group $(p<0.05)$. Moreover, Gly-induced oxidative stress in rat kidney, indicated by reduced TAC, SOD and GSH levels, compared to the control group $(p<0.05)$.

Meanwhile, sildenafil treatment produced a significant decrease in lipid peroxidation, confirmed by the reduced MDA level and had no significant effect on nitrosative stress, indicated by the slight decrease in NO level, compared to the Gly group. Additionally, Sild treatment showed a notable antioxidant effect, as it induced a significant elevation in the level of TAC and SOD with some amelioration in the level of GSH, compared to the Gly group.

Furthermore, DATS treatment in a dose related manner produced a significant gradual decrease in lipid peroxidation and nitrosative stress, indicated by the reduced MDA and NO levels, compared to the Gly group. In addition, the higher dose of DATS $(20 \mathrm{mg} / \mathrm{kg})$ showed a remarkable antioxidant effect, indicated by the elevation in TAC, compared to the Gly group, the elevation in SOD activity, compared to all treated groups and the elevation in GSH level compared to Gly, and DATS 10 treated groups. Whereas, treatment with the lower dose of DATS $(10 \mathrm{mg} / \mathrm{kg}$ ) produced less powerful antioxidant effect, indicated by a lesser significant increase in TAC and no effect on the SOD activity and GSH level compared to the Gly group.

\subsection{Effect on NRF-2/HO-1 pathway}

The expression levels of the antioxidant marker; NRF-2 and its downstream target $\mathrm{HO}-1$, were evaluated in renal homogenates. Treatment with sildenafil or DATS $(20 \mathrm{mg} / \mathrm{kg})$ significantly enhanced NRF-2 and HO1 expression levels in the kidney, compared to Gly and control groups $(p<0.05)$. Whereas, the lower dose of DATS (10 mg/kg) produced a less remarkable elevation in HO-1 expression level, compared to Gly and 
control groups $(p<0.05)$, and showed insignificant increase in NRF-2 expression level, compared to Gly group. (Fig. 4)

\subsection{Effect on the inflammatory mediators (IL-1 $\beta$ and IL-6)}

As shown in Fig. 5, Gly induced a significant increase in the expression levels of renal inflammatory cytokines (IL-1 $\beta$, and IL-6), compared to the control value $(\mathrm{p}<0.05)$. Meanwhile, levels of expression of these inflammatory cytokines were significantly reduced in all groups treated with DATS (10 and 20 $\mathrm{mg} / \mathrm{kg}$ ), compared to Gly group. Whereas, Sild treated group produced no significant variation in the levels of expression of IL-1 $\beta$ and IL-6, compared to the Gly group.

\subsection{Effect on NF-kB inflammatory pathway}

Acute kidney injury involves the release of several inflammatory mediators. TNF-a is an inflammatory mediator that stimulates many inflammatory pathways, including NF-kB, which is the main transcription factor that triggers inflammatory responses. COX-2 is an inducible enzyme participates in inflammatory process, as it leads to the production of inflammatory prostaglandins.

Renal sections in (Fig. 6A, 7A, 8A), were immunostained to detect the expression levels of TNF-a, NF-KB p65 and COX-2. Gly treatment significantly enhanced the expression of these inflammatory markers, as it showed strong positive nuclear staining in renal tubules (black and yellow arrows) and glomeruli (black arrowheads), and a high expression score (Fig. 6B, 7B, 8B), compared to the control groups.

Treatment with sildenafil or DATS $(20 \mathrm{mg} / \mathrm{kg}$ ) significantly attenuated TNF- $\mathrm{a} / \mathrm{NF}-\mathrm{kB}$ activation, and COX2 enzyme expression, indicated with a decrease in positive nuclear staining in renal tubules (black and yellow arrows) and glomeruli (black arrowheads), and expression score, compared to Gly group. Whereas, the lower dose of DATS $(10 \mathrm{mg} / \mathrm{kg})$ produced a significant decline in positive staining and expression score of COX-2, with a partial insignificant lowering in positive staining and expression score of TNF-a and NF-kB, compared to Gly group.

\subsection{Effect on apoptosis (BAX/BCL-2)}

Apoptosis can be mediated through BAX, which then recruit effector caspases to cause apoptotic death, however this mechanism can be counterbalanced by anti-apoptotic BCL-2 protein.

Immunostained renal sections against Bax (Fig. 9A), showed marked positive staining appears in renal tubules (yellow arrows) of Gly treated group. The positive expression markedly decreased in renal tubules (yellow arrows) of treated groups with Sild and DATS $(10 \mathrm{mg} / \mathrm{kg})$, and returned negative in treated group with DAT $(20 \mathrm{mg} / \mathrm{kg})$, when compared to Gly group.

Immunostained renal sections against BCL-2 (Fig. 9B), showed marked decrease of positive expression against BCL-2 appears in renal tubules (yellow arrows) of Gly treated group. The positive expression notably increased in renal tubules (yellow arrows) of treated groups with Sild and DATS $(10 \mathrm{mg} / \mathrm{kg}$ ), and 
produced the most remarkable increase in treated group with DATS $(20 \mathrm{mg} / \mathrm{kg})$, when compared to Gly group.

Moreover, the Bax/BCL-2 ratio revealed a marked increase in Gly treated group, compared to the normal values. Treatment with Sild and DATS $(10 \mathrm{mg} / \mathrm{kg}$ ) reversed this abnormal change in Bax/BCL-2 ratio, compared to Gly group. Meanwhile, treatment with DATS $(20 \mathrm{mg} / \mathrm{kg})$ produced the most significant decrease in this ratio compared to Gly value (Fig. 9C).

\subsection{Effect on renal histology}

As shown in Fig. 10A, histology for renal sections showed normal tubules, glomeruli and interstitial tissue in control group. Meanwhile, diffuse acute tubular necrosis (black arrow), tubular dilation with cast formation (yellow arrow) were observed in Gly group. Focal tubular necrosis (black arrow) with mild tubular dilation and cast formation (yellow arrow) were observed in Sild group. Congestion (red arrows) with degenerated glomeruli (white arrow) was observed in DATS 10 treated group and pictures of renal cortices showed the most improvement in DATS 20 treated group. Renal injury score values indicated that all drug treated groups showed significant near normal improvement in renal histology, compared to Gly and control groups (Fig. 10B)

\section{Discussion}

Glycerol-induced rhabdomyolysis in rats establishes adjacent alikeness to rhabdomyolysis in humans, with respect to histopathological lineaments and cytokine profile (Song, Kim et al. 2020).

This study showed that Gly-induced renal injury was proved by elevation in levels of serum and urine creatinine, urine albumin, urine T. protein and BUN, in addition to irregular histological profile: acute tubular necrosis, brush border loss, tubular dilation with cast formation. These abnormalities might be ascribed to initiation of the inflammation cascade and generation of ROS, leading to the disruption and degeneration of structural integrity in kidney cells, and consequently, renal damage, as mentioned with numerous earlier studies (Gu, Yang et al. 2014, Li, Xu et al. 2019). Moreover, in this research, Gly caused a remarkable increase in mortality, and RSI, which is expected to be a consequence of the above mentioned proteinuria, albuminuria and renal damage.

Meanwhile, the studied drugs in order of potency: DATS $20 \mathrm{mg}$, followed by DATS $10 \mathrm{mg}$, then lastly, sildenafil maintained the overall body health. They produced significant alleviation in Gly-induced renal injury, histopathological manifestations and RSI, as verified by the significant decrease in all elevated renal function tests, retention of normal histological architecture and improvement of survival.

Furthermore, intramuscular injection of glycerol augmented levels of CK and its isoenzyme CK-MB, leading to general skeletal muscle injury and release of myoglobin into blood that in turn caused AKI (Holt and Moore 2000). Treatment with sildenafil and DATS (20 mg/kg) achieved improvement in Gly-induced acute muscle injury, while the lower dose of DATS $(10 \mathrm{mg} / \mathrm{kg})$ was insufficient to alleviate muscle injury. 
This study indicated for the first time that sildenafil and DATS could be muscle protective drugs, through reversal of the elevated serum levels of total CK and CK-MB in rhabdomyolysis model.

Many mechanisms contribute to glycerol-induced AKI, including: oxidative stress, renal vasoconstriction, inflammation and intra-tubular cast formation. Oxidative stress showed a serious role in the development of RIAKI, as it leads to the damage of renal tubular cells, which subsequently prompts the liberation of cytokines and chemokines, and triggers leukocyte stimulation (Koupenova and Ravid 2013)

In the current study, glycerol-induced oxidative stress was expressed by the elevated renal MDA and NO levels and the decreased renal antioxidant enzymes: SOD and GSH, in addition to the overall renal TAC. The results showed that DATS $(20 \mathrm{mg} / \mathrm{kg})$ was able to reverse all Gly-induced oxidant/antioxidant disturbances, therefore owns a remarkable antioxidant activity, which signifies its potential to prevent oxidative and nitrosative damage, while the lower dose of DATS $(10 \mathrm{mg} / \mathrm{kg})$ had a lesser pronounced antioxidant effect. These outcomes are in convenience with a previous study, which documented that DATS had a protective effect against oxidative stress (Prabu and Sumedha 2014), in addition to another study, which reported that garlic polysulfide decreased ROS generation and alleviated neutrophil infiltration in the kidney (Ko et al., 2017; Miltonprabu et al., 2017).

The ability of Sildenafil to improve glycerol-induced elevation in free radicals is confirmed in our study via significant elevation of antioxidants, and reduction of lipid peroxidation to fight free radicals. This was reliable with the outcomes of previous studies, which confirmed its antioxidant effects and presented that Sild decreased malondialdehyde in several experimentally-induced kidney injuries in rats (Cadirci, Halici et al. 2011, Küçük, Yucel et al. 2012, Morsy, Ibrahim et al. 2014). Previous reports denoted that Sild was able to increase GSH (Beheshtian, Salmasi et al. 2008), superoxide dismutase (Sanders 2020) and TAC (lordache, Docea et al. 2020) activities. Meanwhile, Sild had no remarked effect on NO level, as it is well known to increase its constitutive levels in several tissues, without producing nitrosative damage. Consequently, sildenafil ( $5 \mathrm{mg} / \mathrm{kg}$ ) can be considered an effective drug in reducing renal oxidative stress, but to a lesser extent than DATS $(20 \mathrm{mg} / \mathrm{kg})$, as presented in this work.

AKI is linked with augmented liberation of cytokines, mainly, TNF-a, IL-1 $\beta$, and IL- 6 that can recruit oxidative flow. TNF-a can excite inflammatory consequences by triggering NF-KB. NF-KB shows a critical role in organizing a lot of genes involved in inflammation (Papaconstantinou, Zeglinas et al. 2014), including COX-2. Increased NF-KB expression, along with increased TNF-a, IL-1 $\beta$, IL-6 and COX-2 expressions were previously confirmed to be directly involved in renal injury (McSweeney, Gadanec et al. 2021).

In this study, glycerol caused elevation in renal levels of the pro-inflammatory cytokines: TNF- $\alpha$, IL-1 $\beta$, and IL-6, in addition to COX-2. This was confirmed in previous reports, which stated that levels of TNF-a, IL-1 $\beta$, IL-6, and COX-2 were elevated in experimentally induced rhabdomyolysis (Geng, Zhang et al. 2014, Homsi, Andreazzi et al. 2015, Ahn, Nam et al. 2016). 
Meanwhile, DATS, in a dose dependent manner, significantly diminished the production of all inflammatory cytokines. The anti-inflammatory effect of DATS was previously reported in several diseases such as cancer (Jurkowska, Wróbel et al. 2017) and ulcerative colitis (Bai, Ouyang et al. 2005), which is in accordance with our results. Sildenafil produced a remarkable decrease in the production of TNF- $\mathrm{a}$ and COX-2, and no variation was observed in the production of other cytokines. This may be due to utility of a single low dose of sildenafil $(5 \mathrm{mg} / \mathrm{kg})$.

It is well known that there is a counterbalance between NRF-2 and NF-KB pathways. NF-KB could directly suppress NRF-2 at the transcriptional level (Wardyn, Ponsford et al. 2015). Otherwise, triggering of NRF2/HO-1 antioxidant signaling weakens inflammatory signaling paths and NF-KB intermediated proinflammatory reactions (Saber, Khalil et al. 2019). Moreover, it has been recognized that NRF-2 activators were created to inhibit IKK/IKB phosphorylation and consequently inhibited NF-kB p65 nuclear translocation, thus, deactivating NF-KB signaling (Dharmani, Leung et al. 2011).

In the present experimental study, treatment with sildenafil and DATS, in a dose dependent manner, achieved improvement in rhabdomyolysis-induced disruption in the balance between NRF-2 and NF-KB, by decreasing the expression of NF-KB and increasing the expression of NRF-2 and HO-1 in RIAKI rats. These consequences suggest that DATS efficiently diminishes inflammation via inhibition of NF-KB signal transduction pathway, which was stated in previous reports as well (Pan, Lin et al. 2016, Yang, Tang et al. 2019). Other studies supported our results and confirmed that DATS activates NRF-2/HO-1 pathway (Kim, Lee et al. 2014, Silva-Islas, Chánez-Cárdenas et al. 2019). Therefore, DATS may be considered as a NF-KB inhibitor, and a NRF-2 activator, possibly by its anti-inflammatory effect, as well as its antioxidant effect. In addition, the resulted modulation of these pathways, proved the antioxidant and anti-inflammatory effects of sildenafil, which was confirmed in previous reports (Kniotek and Boguska 2017, Maziero Alves, Aires et al. 2021).

The augmented apoptosis level in rhabdomyolysis, leads to acute kidney injury, and the significant raise in the level of apoptotic Bax is well known to be correlated to the anti-apoptotic BCL-2 protein (Wang, Zhang et al. 2011). In this study, sildenafil and DATS, in a dose-dependent mode, decreased the ratio of Bax/BCL-2 as noticed by immunolabeling. This is coincided with a former study, which confirmed that DATS protected against apoptosis during myocardial ischemia-reperfusion injury in diabetic rats $(\mathrm{Yu}, \mathrm{Li}$ et al. 2017).

\section{Conclusion}

In conclusion, DATS, in a dose-related mode, improved rhabdomyolysis-induced AKI and its inflammatory signs, as indicated by histopathological and immunohistochemical assessment. In addition, DATS was proved to be an initiator of NRF-2/HO-1, and an inhibitor of NF-KB and apoptotic signaling pathways, resulting in depression of inflammatory mediators, and elevation of kidney anti-oxidant defense machinery. Consequently, DATS proved its muscle and renal protective effect in rats. Meanwhile, sildenafil, to a less pronounced extent, followed the same mechanistic pathway and proved its protective 
effect. Therefore, we assume that DATS can be a promising natural candidate for healing acute muscle and kidney injuries in humans.

\section{Abbreviations}

AKI, acute kidney injury; BAX, BCL-2 associated x protein; BCL-2, B-cell lymphoma-2; BUN, blood urea nitrogen; $\mathrm{CKI}$, chronic kidney injury; CK, creatine kinase; CK-MB, creatine kinase-MB; $\mathrm{Cr}$, creatinine; DATS, diallyl trisulphides; ELISA, sandwich enzyme-linked immunosorbent assay; Gly, glycerol; GSH, reduced glutathione; HO-1, heme oxygenase-1; HRP, streptavidin horseradish peroxidase; IL, interleukin; MDA, malondialdehyde; NF-kB, nuclear transcription factor kappa B; NO, nitric oxide; NRF-2, nuclear factor erythroid-2 related factor-2; PBS, phosphate buffer saline; PDE-5, phosphodiesterase-5; RIAKI, rhabdomyolysis-induced acute kidney injury; RSI, renal somatic index; SOD, superoxide dismutase; Sild, Sildenafil; TAC, total antioxidant capacity; TNF-a, tumor necrosis factor-alpha.

\section{Declarations}

\section{Acknowledgments}

The authors kindly thank Dr. Walla F. Awadin, Professor of Pathology at Mansoura University's Faculty of Veterinary Medicine, who assisted in completing the histopathological and immunohistochemical section of this research.

\section{Author Contributions}

Ghada S. El-Tanbouly contributed to conception, conducted the histological examinations, handled animals, provided softwares, and conducted statistical analysis. Ghada S. El-Tanbouly and Rania M. Khalil, contributed to data analysis and discussion, manuscript synthesis, wrote and revised the manuscript and designed the graphical abstract. All authors have read and approved the manuscript for publication.

\section{Ethical approval}

The study was conducted according to the ARRIVE guidelines of the National Centre for the Replacement, Refinement and Reduction of Animals in Research (NC3Rs). All procedures were permitted by the Institutional Animal Care and Use Committee (IACUC) at the Faculty of Pharmacy, Delta University for Science and Technology, Gamasa, Egypt, with approval number (FPDU 150321/5).

\section{Consent for publication}

Not applicable. 


\section{Consent to participate}

Not applicable.

\section{Availability of data and materials}

The datasets analyzed during the current study are available from the corresponding author on reasonable request.

\section{Conflicts of Interest Statement}

The authors declare no conflict of interest.

\section{Funding}

This research did not receive any specific grant from funding agencies in the public, commercial, or notfor-profit sectors.

\section{References}

1. Ahn, H. J., O. H. Nam, H. S. Lee, E. C. Kim, N. Cohenca and S. C. Choi (2016). "Expression of inflammatory cytokines and MMPs on replanted teeth at different extra-alveolar time: an ex vivo and in vivo study." Int J Paediatr Dent 26(4): 301-309.

2. Bai, A. P ,.Q. Ouyang and R. W. Hu (2005). "Diallyl trisulfide inhibits tumor necrosis factor-alpha expression in inflammed mucosa of ulcerative colitis." Dig_Dis Sci 50(8): 1426-1431.

3. Beheshtian, A., A. H. Salmasi, S. Payabvash, S. Kiumehr, B. Ghazinezami, S. Rahimpour, S. M. Tavangar and A. R. Dehpour (2008). "Protective effects of sildenafil administration on testicular torsion/detorsion damage in rats." World J Urol 26(2): 197-202.

4. Behiry, S., A. Rabie, M. Kora, W. Ismail, D. Sabry and A. Zahran (2018). "Effect of combination sildenafil and gemfibrozil on cisplatin-induced nephrotoxicity; role of heme oxygenase-1." Ren Fail 40(1): 371-378.

5. Cadirci, E., Z. Halici, F. Odabasoglu, A. Albayrak, E. Karakus, D. Unal, F. Atalay, I. Ferah and B. Unal (2011). "Sildenafil treatment attenuates lung and kidney injury due to overproduction of oxidant activity in a rat model of sepsis: a biochemical and histopathological study." Clin Exp Immunol 166(3): 374-384.

6. Caro-Gómez, L. A., J. L. Rosas-Trigueros, E. Mixcoha, J. L. Vique-Sánchez, H. Gasperin-Sánchez, C. G. Benítez-Cardoza and A. Zamorano-Carillo (2019). "Exploring the Conformational Space of Bcl-2 
Protein Variants: Dynamic Contributions of the Flexible Loop Domain and Transmembrane Region." Molecules 24.(21)

7. Dharmani ,P., P. Leung and K. Chadee (2011). "Tumor necrosis factor-a and Muc2 mucin play major roles in disease onset and progression in dextran sodium sulphate-induced colitis." PLoS One 6(9): e25058.

8. Dias, A. T., B. P. Rodrigues, M. L. Porto, A. L. Gava, C. M .Balarini, F. P. Freitas, Z. Palomino, D. E. Casarini, B. P. Campagnaro, T. M. Pereira, S. S. Meyrelles and E. C. Vasquez (2014). "Sildenafil ameliorates oxidative stress and DNA damage in the stenotic kidneys in mice with renovascular hypertension." J Transl Med 12: 35.

9. Geng, X., Y. Wang, Q. Hong, J. Yang, W. Zheng, G. Zhang, G. Cai, X. Chen and D. Wu (2015). "Differences in gene expression profiles and signaling pathways in rhabdomyolysis-induced acute kidney injury." Int J Clin Exp Pathol 8(11): 14087-1.4098

10. Geng, Y., L. Zhang, B. Fu, J. Zhang, Q. Hong, J. Hu, D. Li, C. Luo, S. Cui, F. Zhu and X. Chen (2014). "Mesenchymal stem cells ameliorate rhabdomyolysis-induced acute kidney injury via the activation of M2 macrophages." Stem Cell Res Ther 5(3): 80.

11. Goldberg, R. and P. Dennen (2008). "Long-term outcomes of acute kidney injury." Adv Chronic Kidney. Dis 15(3): 297-307.

12. Gu, H., M. Yang, X. Zhao, B. Zhao, X. Sun and X. Gao (2014). "Pretreatment with hydrogen-rich saline reduces the damage caused by glycerol-induced rhabdomyolysis and acute kidney injury in rats." $\underline{\mathrm{J}}$ Surg Res 188(1): 243-249.

13. Gurbuz, N., A. Kol, T. Ipekci, E. Ates, A. Baykal and M. F. Usta (2015). "Chronic administration of sildenafil improves erectile function in a rat model of chronic renal failure." Asian J Androl 17(5): 797801.

14. Havasi, A. and S. C. Borkan (2011). "Apoptosis and acute kidney injury." Kidney International 80(1): 29-40.

15. Holt, S. and K. Moore (2000). "Pathogenesis of renal failure in rhabdomyolysis: the role of myoglobin ".Exp Nephrol 8(2): 72-76.

16. Homsi, E., D. D. Andreazzi, J. B. Faria and P. Janino (2015). "TNF-a-mediated cardiorenal injury after rhabdomyolysis in rats." Am J Physiol Renal Physiol 308(11): F1259-1267.

17. Huang, R. S., J. J. Zhou, Y. Y. Feng, M. Shi, F. Guo ,S. J. Gou, S. Salerno, L. Ma and P. Fu (2017). "Pharmacological Inhibition of Macrophage Toll-like Receptor 4/Nuclear Factor-kappa B Alleviates Rhabdomyolysis-induced Acute Kidney Injury." Chin Med J (Engl). 130(18): 2163-2169.

18. Iordache, A. M., A. O. Docea, A. M. Buga, O. Zlatian, M. E. Ciurea, O. C. Rogoveanu, F. Burada, S. Sosoi, R. Mitrut, C. Mamoulakis, D. Albulescu, R. C. Vasile, A. Tsatsakis and D. Calina (2020). "Sildenafil and tadalafil reduce the risk of contrast-induced nephropathy by modulating the oxidant/antioxidant balance in a murine model." Food Chem Toxicol 135: 111038.

19. Jurkowska, H., M. Wróbel, M. Kaczor-Kamińska and E. Jasek-Gajda (2017). "A possible mechanism of inhibition of U87MG and SH-SY5Y cancer cell proliferation by diallyl trisulfide and other aspects of 
its activity." Amino Acids 49(11): 1855-1866.

20. Khames, A., M. M. Khalaf, A. M. Gad and O. M. Abd El-Raouf (2017). "Ameliorative effects of sildenafil and/or febuxostat on doxorubicin-induced nephrotoxicity in rats." Eur J Pharmacol 805: 118-124.

21. Kim, S., H. G. Lee, S. A. Park, J. K. Kundu, Y. S. Keum, Y. N. Cha, H. K. Na and Y. J. Surh (2014). "Keap1 cysteine 288 as a potential target for diallyl trisulfide-induced Nrf2 activation." PLoS One 9(1): e85984.

22. Kniotek, M. and A. Boguska (2017). "Sildenafil Can Affect Innate and Adaptive Immune System in Both Experimental Animals and Patients." J Immunol Res 2017: 4541958.

23. Koupenova, M. and K. Ravid (2013). "Adenosine, adenosine receptors and their role in glucose homeostasis and lipid metabolism." $\underline{\mathrm{J} \text { Cell Physiol. }}$

24. Küçük, A., M. Yucel, N. Erkasap, M. Tosun, T. Koken, M. Ozkurt and S. Erkasap (2012). "The effects of PDE5 inhibitory drugs on renal ischemia/reperfusion injury in rats." Mol Biol Rep 39(10): 9775-9782.

25. Li, Y. F., B. Y. Xu, R .An, X. F. Du, K. Yu, J. H. Sun, G. H. Zhang, W. Wang, L. P. An and G. L. Wu (2019). "Protective effect of anisodamine in rats with glycerol-induced acute kidney injury." BMC Nephrol 20(1): 223.

26. Liang, J. J., H. R. Li, Y. Chen, C. Zhang, D. G. Chen, Z. C .Liang, Y. Q. Shi, L. L. Zhang, L. Xin and D. B. Zhao (2019). "Diallyl Trisulfide can induce fibroblast-like synovial apoptosis and has a therapeutic effect on collagen-induced arthritis in mice via blocking NF-KB and Wnt pathways." Int Immunopharmacol 71.132-138:

27. Ma, H. B., S. Huang, X. R. Yin, Y. Zhang and Z. L. Di (2014). "Apoptotic pathway induced by diallyl trisulfide in pancreatic cancer cells." World J Gastroenterol 20(1): 193-203.

28. Maziero Alves, G., R. Aires, V. de Souza Santos, L. Zambom Côco ,B. Peters, A. de Leone Evangelista Monteiro Assis, B. Ramos Athaydes, F. Gobbi Amorim, B. Valentim Nogueira, R. C. de Ribeiro Gonçalves, S. Dos Santos Meyrelles, T. Melo Costa Pereira and B. Prandi Campagnaro (2021). "Sildenafil attenuates nonsteroidal anti-inflammatory-induced gastric ulceration in mice via antioxidant and antigenotoxic mechanisms." Clin Exp Pharmacol Physiol 48(3): 401-411.

29. McSweeney, K. R., L. K. Gadanec, T. Qaradakhi, B. A. Ali, A. Zulli and V. Apostolopoulos (2021). "Mechanisms of Cisplatin-Induced Acute Kidney Injury: Pathological Mechanisms, Pharmacological Interventions, and Genetic Mitigations." Cancers (Basel). 13.(7)

30. Miltonprabu, S., N. C. Sumedha and P. Senthilraja (2017). "Diallyl trisulfide, a garlic polysulfide protects against As-induced renal oxidative nephrotoxicity, apoptosis and inflammation in rats by activating the Nrf2/ARE signaling pathway." Int Immunopharmacol 50: 107-120.

31. Mohey, V., M. Singh, N. Puri, T. Kaur, D. Pathak and A. P. Singh (2016). "Sildenafil obviates ischemiareperfusion injury-induced acute kidney injury through peroxisome proliferator-activated receptor $Y$ agonism in rats." J Surg Res 201(1): 69-75.

32. Morsy, M. A., S. A. Ibrahim, E. F. Amin, M. Y. Kamel, R. A. Rifaai and M. K. Hassan (2014). "Sildenafil Ameliorates Gentamicin-Induced Nephrotoxicity in Rats: Role of iNOS and eNOS." J Toxicol 2014: 
489382.

33. Pan, Y., S. Lin, R. Xing, M. Zhu, B. Lin, J. Cui, W. Li, J. Gao, L. Shen, Y. Zhao, M. Guo, J. M. Wang, J. Huang and Y. Lu (2016). "Epigenetic Upregulation of Metallothionein 2A by Diallyl Trisulfide Enhances Chemosensitivity of Human Gastric Cancer Cells to Docetaxel Through Attenuating NF-kB Activation." Antioxid Redox Signal 24(15): 839-854.

34. Panizo, N., A. Rubio-Navarro, J. M. Amaro-Villalobos, J. Egido and J. A. Moreno (2015). "Molecular Mechanisms and Novel Therapeutic Approaches to Rhabdomyolysis-Induced Acute Kidney Injury." Kidney_Blood Press Res 40(5): 520-532.

35. Papaconstantinou, I., C. Zeglinas, M. Gazouli, K. Nastos, A. Yiallourou, A. Papalois and C. Tzathas (2014). "The impact of peri-operative anti-TNF treatment on anastomosis-related complications in Crohn's disease patients. A critical review." J Gastrointest Surg 18(6): 1216-1224.

36. Prabu, S. M. and N. C. Sumedha (2014). "Ameliorative effect of diallyl trisulphide on arsenic-induced oxidative stress in rat erythrocytes and DNA damage in lymphocytes." $\underline{\mathrm{J} \text { Basic Clin Physiol }}$ Pharmacol 25(2): 181-197.

37. Qiu, L. Q., R. Sinniah and I. H. H. S (2004). "Downregulation of Bcl-2 by podocytes is associated with progressive glomerular injury and clinical indices of poor renal prognosis in human IgA nephropathy." J Am Soc Nephrol 15(1): 79-90.

38. Saber, S., R. M. Khalil, W. S. Abdo, D. Nassif and E. El-Ahwany (2019). "Olmesartan ameliorates chemically-induced ulcerative colitis in rats via modulating NFKB and Nrf-2/HO-1 signaling crosstalk." Toxicol Appl Pharmacol 364: 120-132.

39. Sanders, O. (2020). "Sildenafil for the Treatment of Alzheimer's Disease: A Systematic Review." $\underline{\mathrm{J}}$ Alzheimers Dis Rep 4(1): 91-106.

40. Savvanis, S., C. Nastos, M. K. Tasoulis, N. Papoutsidakis, M. Demonakou, I. Karmaniolou, N. Arkadopoulos, V. Smyrniotis and K. Theodoraki (2014). "Sildenafil attenuates hepatocellular injury after liver ischemia reperfusion in rats: a preliminary study." Oxid Med Cell Longev 2014: 161942.

41. Shang, A., S. Y. Cao, X. Y. Xu, R. Y. Gan, G. Y. Tang, H. Corke, V. Mavumengwana and H. B. Li (2019). "Bioactive Compounds and Biological Functions of Garlic (Allium sativum L.)." Foods 8.(7)

42. Sharma, A. K., A. Kaur, J. Kaur, G. Kaur, A. Chawla, M. Khanna, H. Kaur, H. Kaur, T. Kaur and A. P. Singh (2021). "Ameliorative Role of Diallyl Disulfide Against Glycerol-induced Nephrotoxicity in Rats." $\underline{\mathrm{J}}$ Pharm Bioallied Sci 13(1): 129-135.

43. Shi, Y., L. Xu, J. Tang, L. Fang, S. Ma, X .Ma, J. Nie, X. Pi, A. Qiu, S. Zhuang and N. Liu (2017). "Inhibition of HDAC6 protects against rhabdomyolysis-induced acute kidney injury." Am J Physiol Renal Physiol 312(3): F502-f515.

44. Shou, Y., N. Li, L. Li, J. L. Borowitz and G. E. Isom (2002). "NF-kappaB-mediated up-regulation of Bcl$\mathrm{X}(\mathrm{S})$ and Bax contributes to cytochrome c release in cyanide-induced apoptosis." J Neurochem 81(4): 842-852.

45. Silva-Islas, C. A., M. E. Chánez-Cárdenas, D. Barrera-Oviedo, A. Ortiz-Plata, J. Pedraza-Chaverri and P. D. Maldonado (2019). "Diallyl Trisulfide Protects Rat Brain Tissue against the Damage Induced by 
Ischemia-Reperfusion through the Nrf2 Pathway." Antioxidants (Basel). 8.(9)

46. Singh, A. P., A. Junemann, A. Muthuraman, A. S. Jaggi, N. Singh, K. Grover and R. Dhawan.(2012) "Animal models of acute renal failure." Pharmacol Rep 64(1): 31-44.

47. Song, S. J., S. M. Kim, S. H. Lee, J. Y. Moon, H. S. Hwang, J. S. Kim, S. H. Park, K. H. Jeong and Y. G. Kim (2020). "Rhabdomyolysis-Induced AKI Was Ameliorated in NLRP3 KO Mice via Alleviation of Mitochondrial Lipid Peroxidation in Renal Tubular Cells." Int J Mol Sci 21.(22)

48. Sureshbabu, A., S. W. Ryter and M. E. Choi (2015). "Oxidative stress and autophagy: crucial modulators of kidney injury." Redox Biol 4: 208-214.

49. Swamy, S. M ,.N. S. Rajasekaran and V. J. Thannickal (2016). "Nuclear Factor-Erythroid-2-Related Factor 2 in Aging and Lung Fibrosis." Am J Pathol 186(7): 1712-1723.

50. Wang, Y. D., L. Zhang, G. Y. Cai, X. G. Zhang, Y. Lv, Q. Hong, S. Z. Shi, Z. Yin, X. F. Liu and X. M .Chen (2011). "Fasudil ameliorates rhabdomyolysis-induced acute kidney injury via inhibition of apoptosis." Ren Fail 33(8): 811-818.

51. Wardyn, J. D., A. H. Ponsford and C. M. Sanderson (2015). "Dissecting molecular cross-talk between Nrf2 and NF-KB response pathways." Biochem Soc Trans 43(4): 621-626.

52. Yang, J., R. Tang, J. Yi, Y. Chen, X. Li, T. Yu and J. Fei (2019). "Diallyl disulfide alleviates inflammatory osteolysis by suppressing osteoclastogenesis via NF-KB-NFATc1 signal pathway." Faseb j 33(6): 7261-7.273

53. Yu, L., S. Li, X. Tang, Z. Li, J. Zhang, X. Xue, J. Han, Y. Liu, Y. Zhang, Y. Zhang, Y. Xu, Y. Yang and H. Wang (2017). "Diallyl trisulfide ameliorates myocardial ischemia-reperfusion injury by reducing oxidative stress and endoplasmic reticulum stress-mediated apoptosis in type 1 diabetic rats: role of SIRT1 activation." Apoptosis 22(7): 942-954.

54. Zhang, F., Y. Zhang, K. Wang, G. Liu, M. Yang, Z. Zhao, S. Li, J. Cai and J. Cao (2016). "Protective effect of diallyl trisulfide against naphthalene-induced oxidative stress and inflammatory damage in mice." Int J Immunopathol Pharmacol 29(2): 205-216.

55. Zhao, W., X. Huang, L. Zhang, X. Yang, L. Wang, Y. Chen, J. Wang and G. Wu (2016). "Penehyclidine Hydrochloride Pretreatment Ameliorates Rhabdomyolysis-Induced AKI by Activating the Nrf2/HO-1 Pathway and Alleviating [corrected] Endoplasmic Reticulum Stress in Rats. The." PLoS One 11(3): e0151158.

\section{Table}

Table 1 is available in the Supplementary Files section.

\section{Figures}



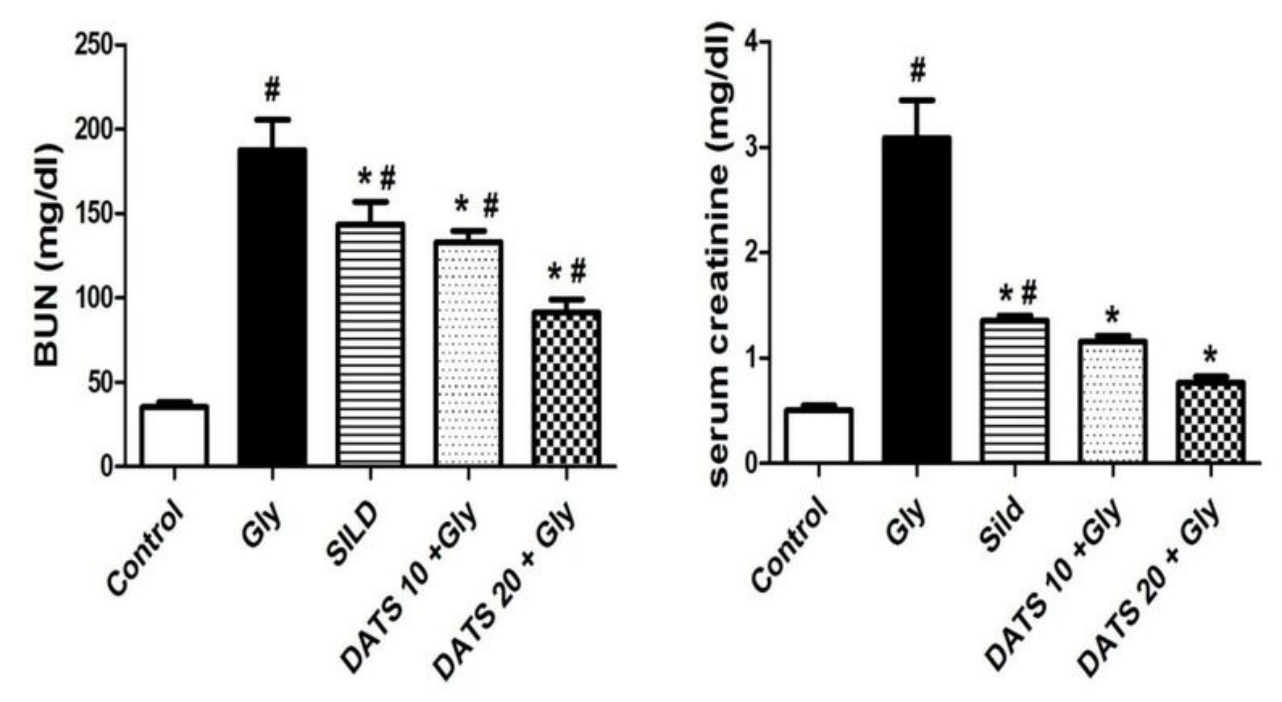

Fig 1
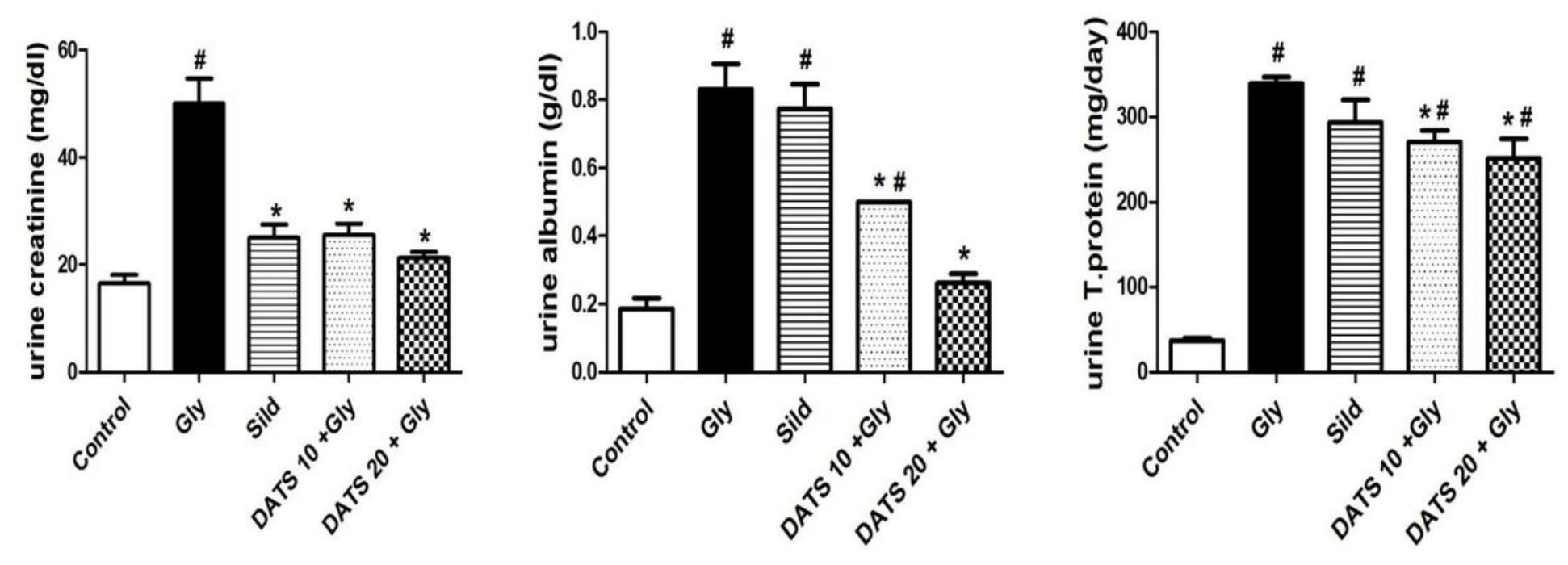

Figure 1

\section{Effect of Sild and DATS treatments on kidney function markers}

Serum levels of BUN, creatinine, in addition to urine concentrations of creatinine, albumin and T.protein were measured in Gly-induced rhabdomyolysis rat model. Data are expressed as mean \pm S.E.M. $(n=8)$. Statistical analysis was performed using one-way ANOVA, followed by Tukey Kramer's post hoc-test, \# * $\mathrm{P}$ $<0.05$ vs. control and Gly groups, respectively, Gly: Glycerol; Sild: Sildenafil; DATS: Diallyl trisulphide; BUN: Blood urea nitrogen; T.protein: total protein 
Fig 2
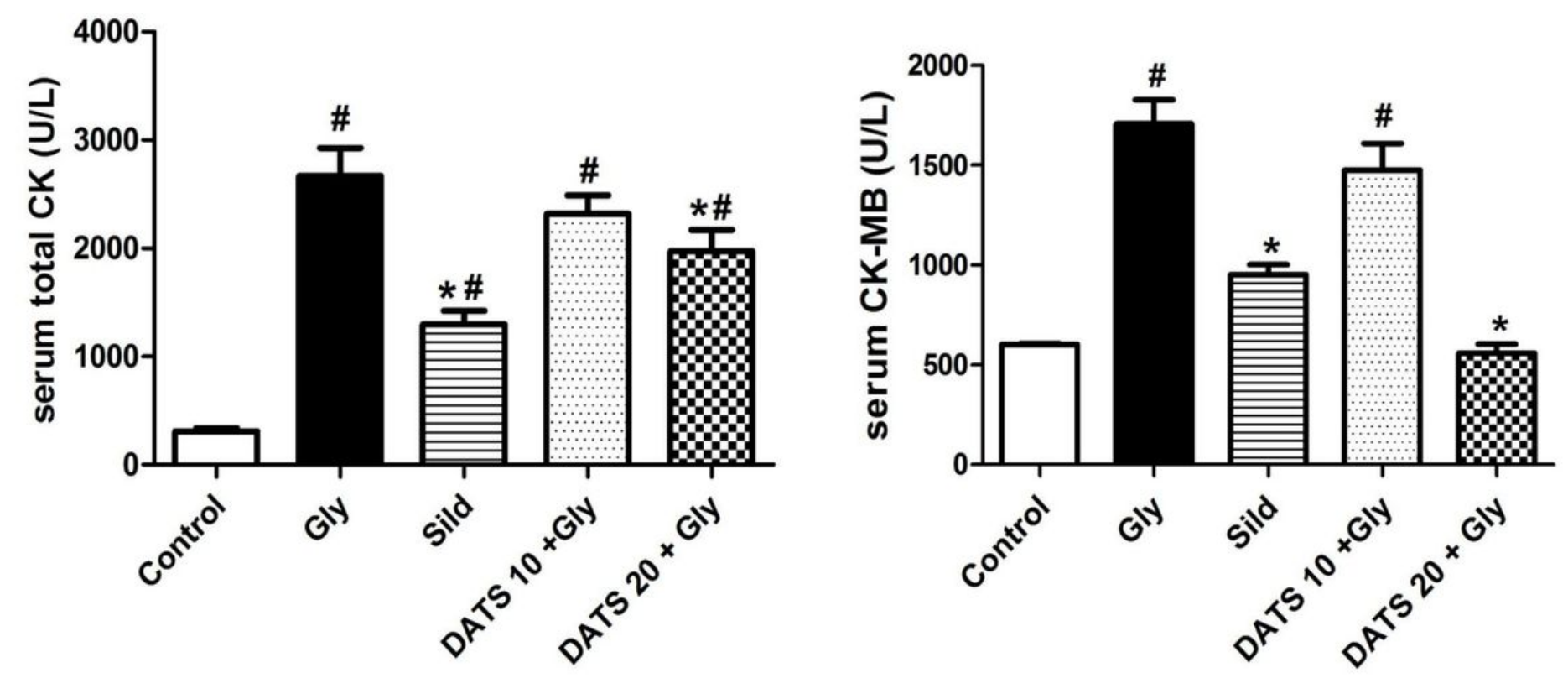

Figure 2

Effect of Sild and DATS treatments on rhabdomyolysis markers

Serum levels of CK and CK-MB are measured in Gly-induced rhabdomyolysis rat model. Data are expressed as mean \pm S.E.M. $(n=8)$. Statistical analysis was performed using one-way ANOVA, followed by Tukey Kramer's post hoc-test, \# * P<0.05 vs. control and Gly groups, respectively, Gly: Glycerol; Sild: Sildenafil; DATS: Diallyl trisulphide; CK: Creatine kinase; CK-MB: Creatine kinase isoenzyme MB 

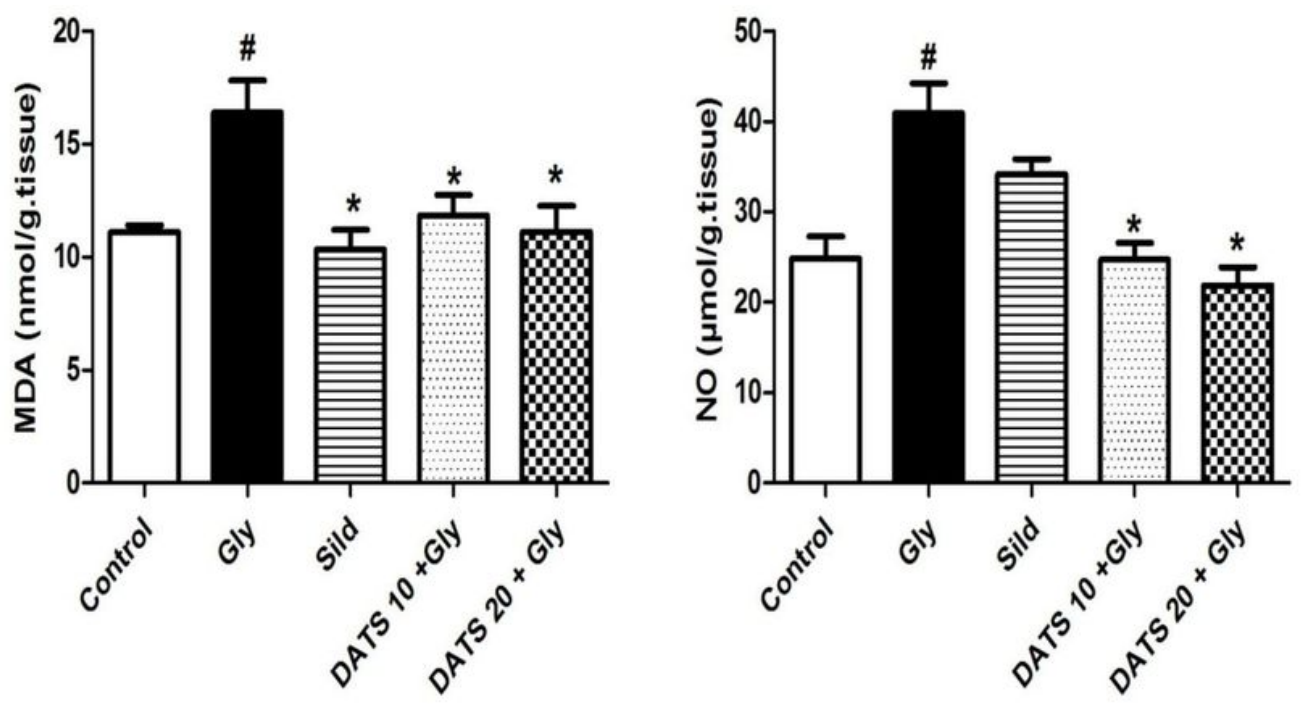

Fig 3
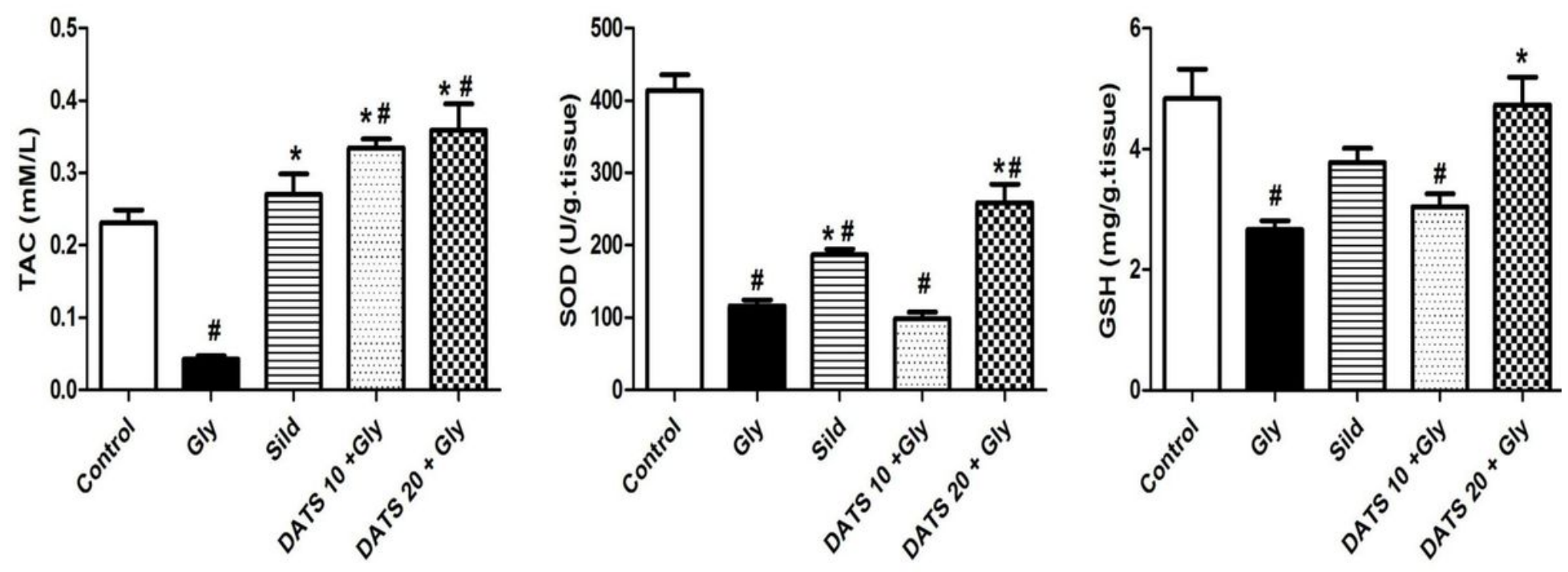

Figure 3

\section{Effect of Sild and DATS treatments on oxidant/antioxidant status}

The lipid peroxidation marker (MDA) and nitrosative stress marker (NO), in addition to the antioxidant markers (TAC, SOD, and GSH), were measured in Gly-induced rhabdomyolysis rat model. Data are expressed as mean \pm S.E.M. $(n=6)$. Statistical analysis was performed using one-way ANOVA, followed by Tukey Kramer's post hoc-test, \# * $\mathrm{P}<0.05$ vs. control and Gly groups, respectively, Gly: Glycerol; Sild: Sildenafil; DATS: Diallyl trisulphide; MDA: Malondialdehyde; NO: Nitric oxide; TAC: Total antioxidant capacity; SOD: Superoxide dismutase; GSH: Reduced glutathione 

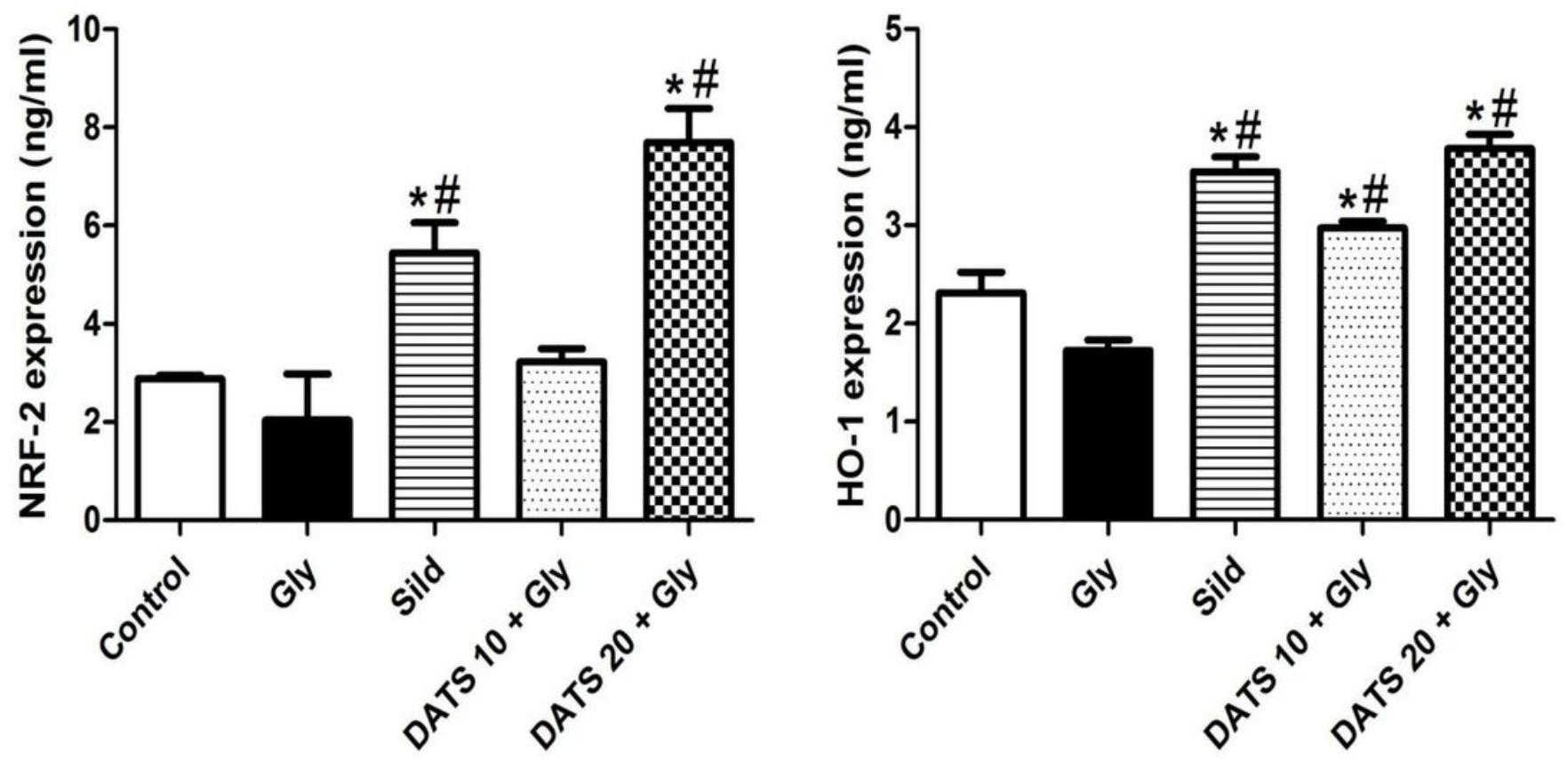

Figure 4

Effect of Sild and DATS treatments on NRF-2/HO-1 pathway

Protein expressions of the antioxidant related markers: NRF-2 and HO-1 are measured in Gly-induced rhabdomyolysis rat model. Data are expressed as mean \pm S.E.M. $(n=5)$. Statistical analysis was performed using one-way ANOVA, followed by Tukey Kramer's post hoc-test, \# ${ }^{*} \mathrm{P}<0.05$ vs. control and Gly groups, respectively, Gly: Glycerol; Sild: Sildenafil; DATS: Diallyl trisulphide; NRF-2: Nuclear factor erythroid-2-related factor-2; HO-1: Heme oxygenase-1 
Fig 5
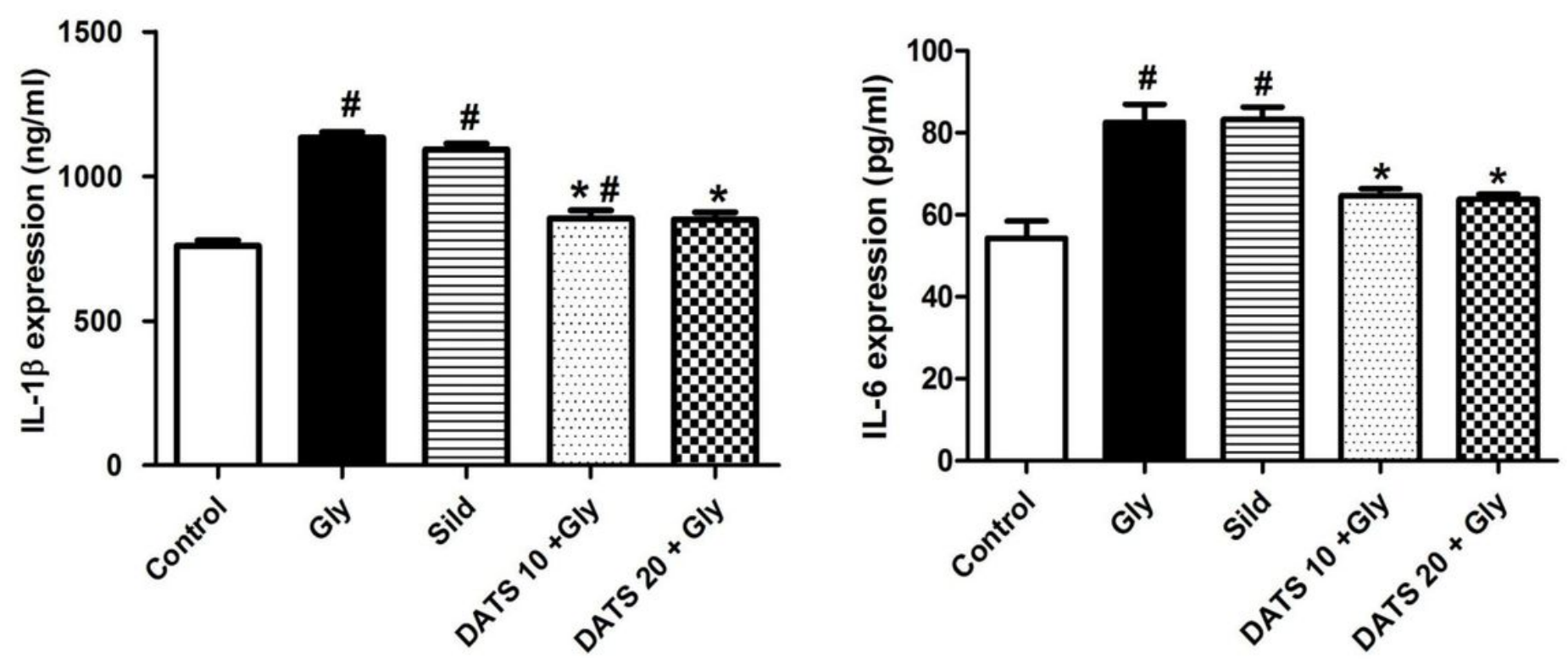

Figure 5

Effect of Sild and DATS treatments on protein expression of the inflammatory mediators (IL-1 $\beta$ and IL-6) in Gly-induced rhabdomyolysis rat model

Data are expressed as mean \pm S.E.M. $(n=5)$. Statistical analysis was performed using one-way ANOVA, followed by Tukey Kramer's post hoc-test, \# $P<0.05$ vs. control and Gly groups, respectively, Gly: Glycerol; Sild: Sildenafil; DATS: Diallyl trisulphide; IL-1ß: Interleukin-1beta; IL-6: Interleukin-6 
Fig 6 A-B

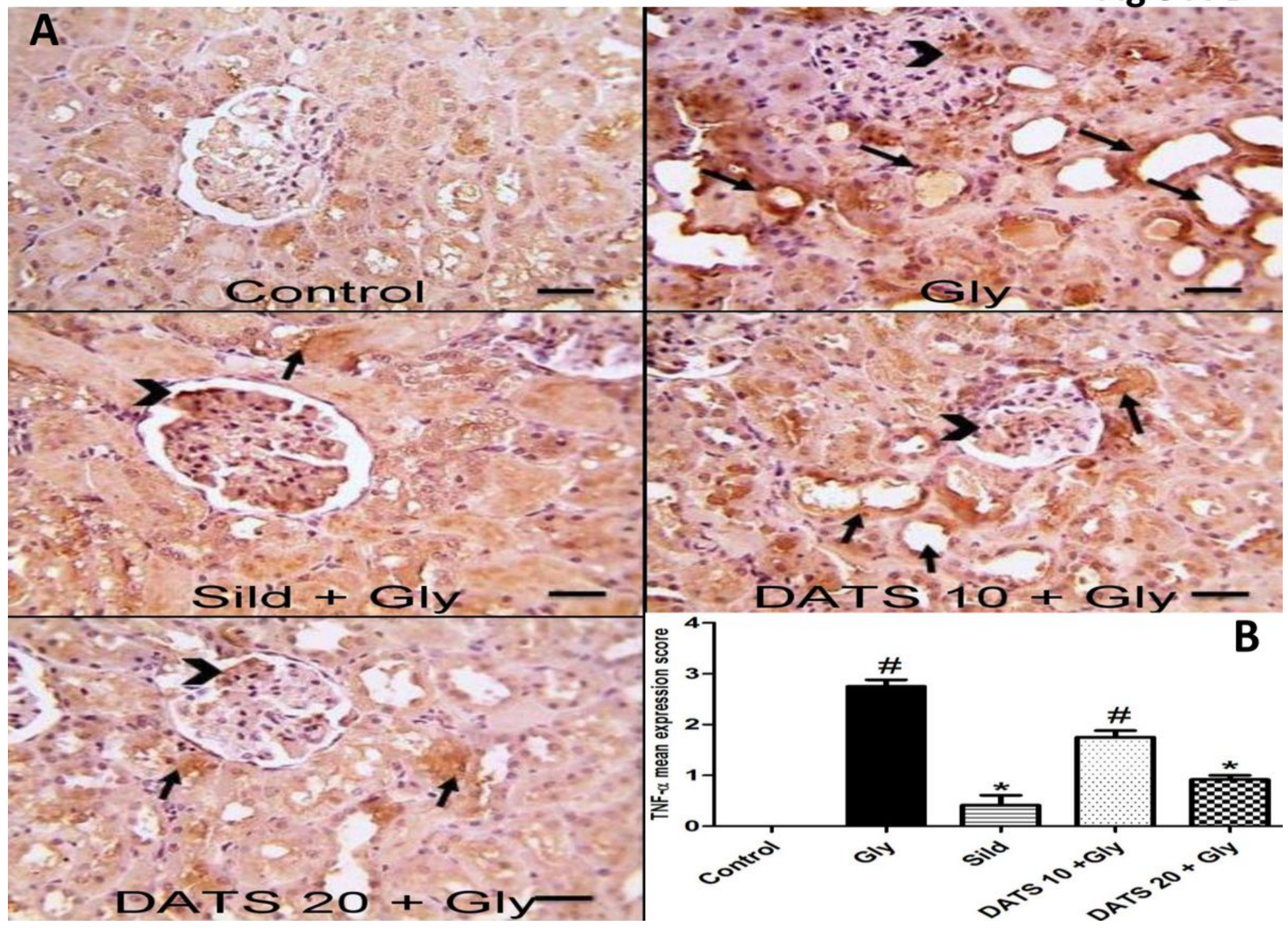

Figure 6

\section{Effect of Sild and DATS treatments on TNF-a expression in Gly-induced rhabdomyolysis rat model}

A: Microscopic pictures of immunohistochemical analysis of TNF-a in renal sections from: normal control, Gly, Sild, DATS 10 and 20 treated groups. IHC counterstained with Mayer's hematoxylin, X: 400, bar: 50 .

B: Analysis of immunostained positive cells to express the mean expression score of TNF- $a$. The data presented are the median $(n=6), \# * p<0.05$ compared with control and Gly groups, respectively using Kruskal-Wallis test followed by Dunn's multiple comparison test, Gly: Glycerol; Sild: Sildenafil; DATS: Diallyl trisulphide; TNF-a: Tumor necrosis factor-alpha 
Fig 7 A-B
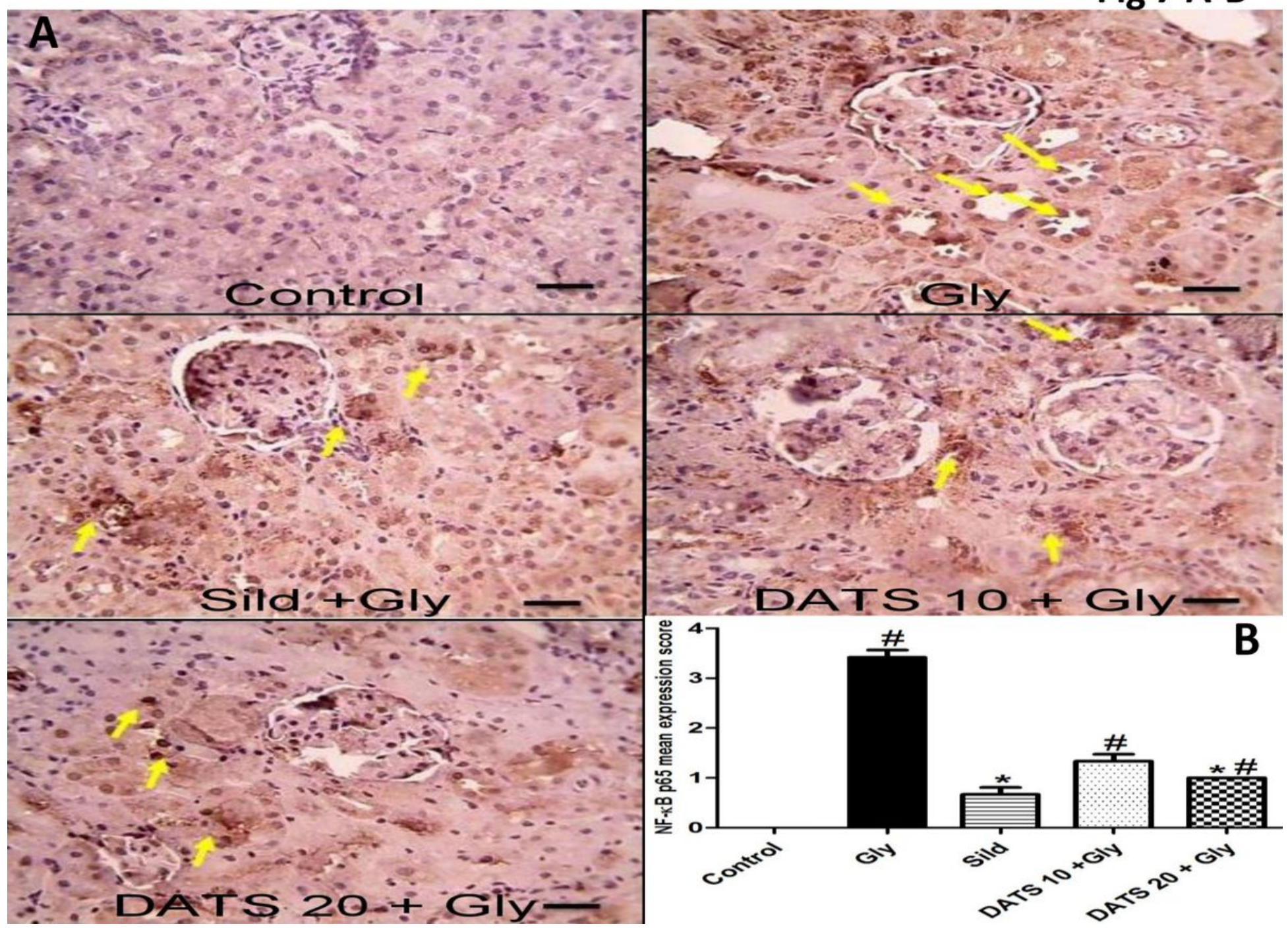

Figure 7

\section{Effect of Sild and DATS treatments on NF-kB expression in Gly-induced rhabdomyolysis rat model}

A: Microscopic pictures of immunohistochemical analysis of NF-kB in renal sections from: normal control, Gly, Sild, DATS 10 and 20 treated groups. IHC counterstained with Mayer's hematoxylin, X: 400, bar: 50 .

B: Analysis of immunostained positive cells to express the mean expression score of NF-KB. The data presented are the median $(n=6), \#$ * $p<0.05$ compared with control and Gly groups, respectively using Kruskal-Wallis test followed by Dunn's multiple comparison test, Gly: Glycerol; Sild: Sildenafil; DATS: Diallyl trisulphide; NF-кB: Nuclear transcription factor kappa B 
Fig 8 A-B

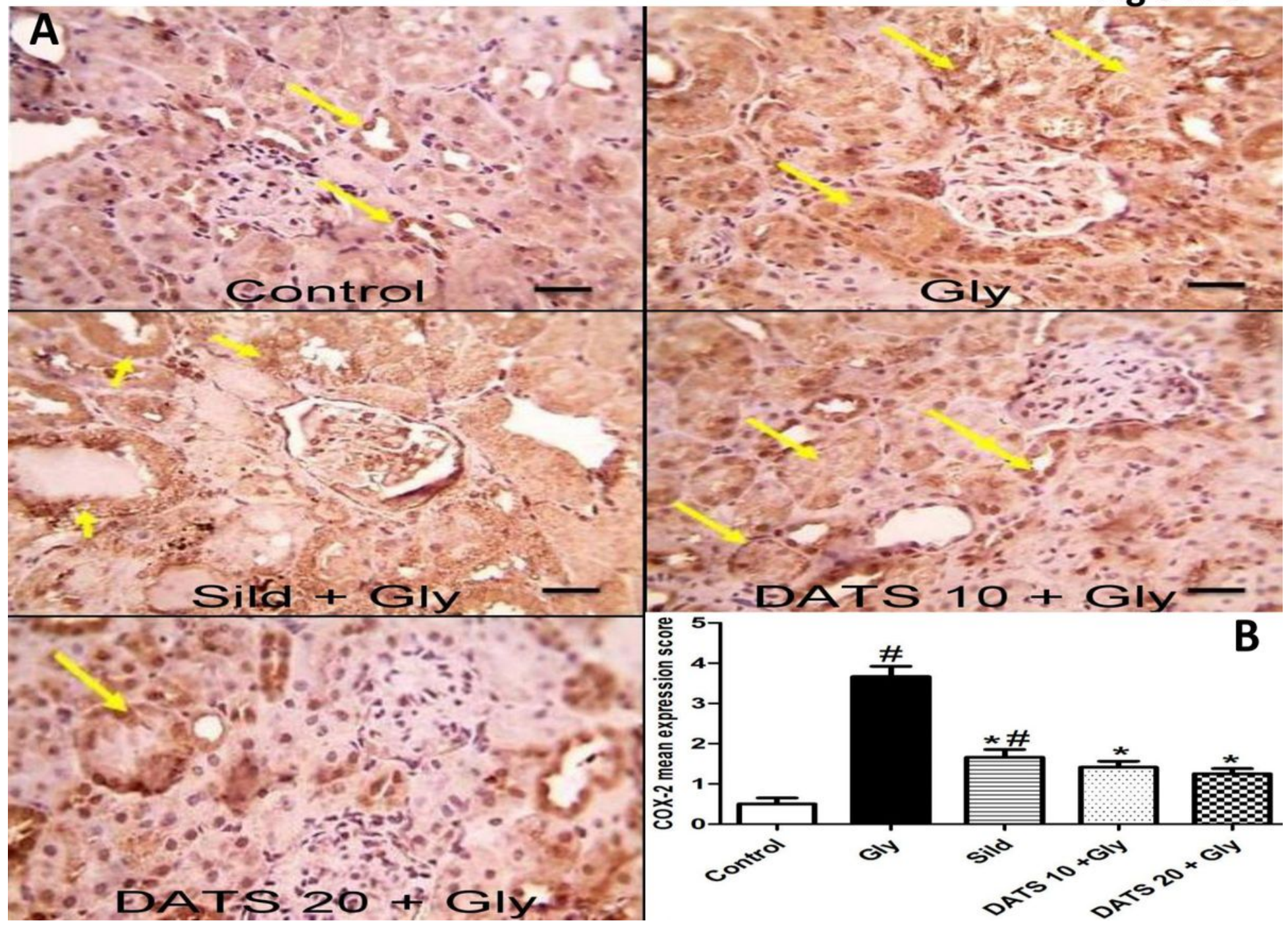

Figure 8

\section{Effect of Sild and DATS treatments on COX-2 expression in Gly-induced rhabdomyolysis rat model}

A: Microscopic pictures of immunohistochemical analysis of COX-2 in renal sections from: normal control, Gly, Sild, DATS 10 and 20 treated groups. IHC counterstained with Mayer's hematoxylin, X: 400, bar: 50 .

B: Analysis of immunostained positive cells to express the mean expression score of COX-2. The data are represented as the median $(n=6), \#$ * $p<0.05$ compared with control and Gly groups, respectively using Kruskal-Wallis test followed by Dunn's multiple comparison test, Gly: Glycerol; Sild: Sildenafil; DATS:

Diallyl trisulphide; COX-2: Cyclooxygenase-2 

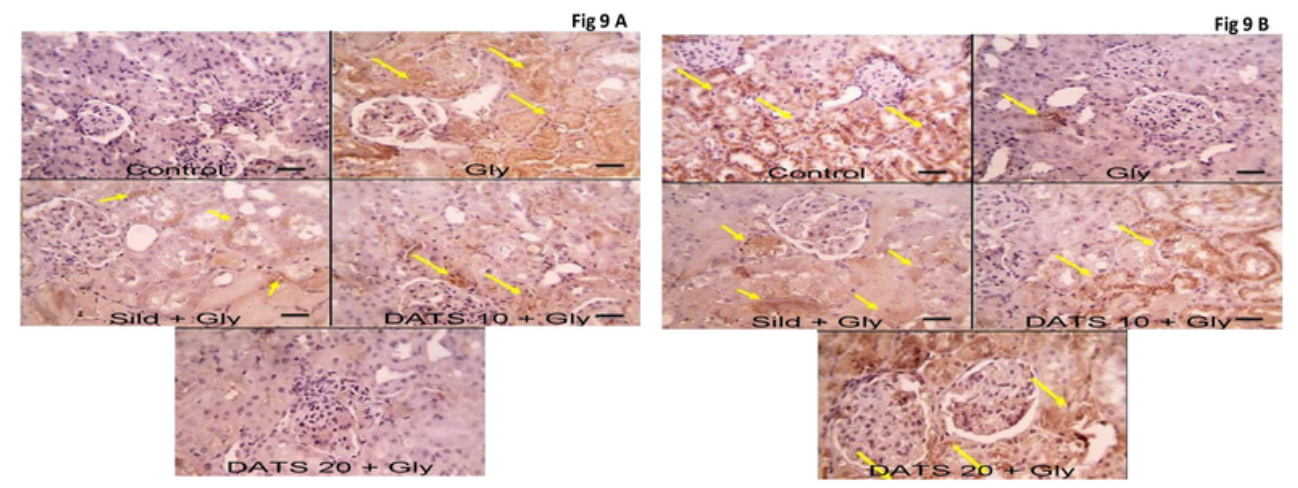

\section{Figure 9}

Effect of Sild and DATS treatments on the apoptotic/antiapoptotic markers (BAX/BCL-2) in Gly-induced rhabdomyolysis rat model

A: Microscopic pictures of immunohistochemical analysis of BAX in renal sections from: normal control, Gly, Sild, DATS 10 and 20 treated groups. IHC counterstained with Mayer's hematoxylin, X: 400, bar: 50 .

B: Microscopic pictures of immunohistochemical analysis of BCL-2 in renal sections from: normal control, Gly, Sild, DATS 10 and 20 treated groups. IHC counterstained with Mayer's hematoxylin, X: 400, bar: 50 .

C: Bar graph showing the effect of Sild and DATS treatments on BAX/BCL-2 ratio. Data are expressed as mean \pm S.E.M. $(n=6)$. Statistical analysis was performed using one-way ANOVA, followed by Tukey Kramer's post hoc-test, \# * $\mathrm{P}<0.05$ vs. control and Gly groups, respectively, Gly: Glycerol; Sild: Sildenafil; DATS: Diallyl trisulphide; BAX: BCL-2 associated x protein; BCL-2: B-cell lymphoma-2 
Fig 10 A-B
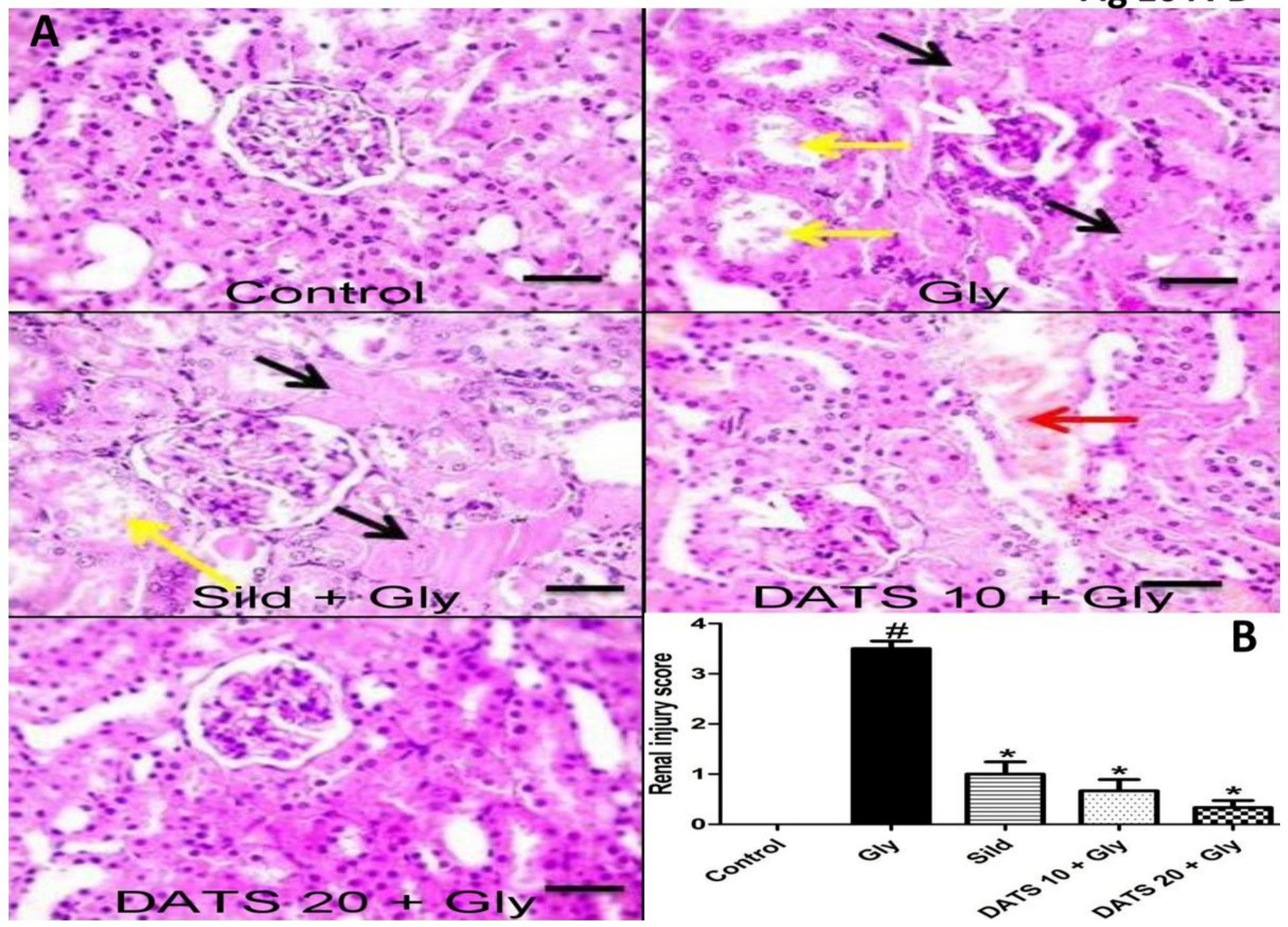

Figure 10

Effect of Sild and DATS treatments on renal histology in Gly-induced rhabdomyolysis rat model

A: Representative photomicrographs of renal sections stained with H\&E, X: 400, bar: 50 .

B: Bar graph showing the effect of Sild and DATS treatments on renal injury score. The data are expressed as the median $(n=6), \# * p<0.05$ compared with control and Gly groups, respectively using Kruskal-Wallis test followed by Dunn's multiple comparison test, Gly: Glycerol; Sild: Sildenafil; DATS: Diallyl trisulphide.

\section{Supplementary Files}

This is a list of supplementary files associated with this preprint. Click to download.

- graphicalabstract.pdf

- Table1.pdf 